\title{
Orthopedic Nursing
}

National Cancer Institute

\section{Source}

National Cancer Institute. Orthopedic Nursing. NCI Thesaurus. Code C20875.

Nursing care with a special emphasis on the care of individuals with orthopaedic-related injuries, diseases, and diagnoses. 